\title{
The Comparison of Duration Endotracheal Tube Insertion Using Aerosol Box and without Aerosol Box in Elective Surgery Patients in the Covid-19 Pandemic
}

\author{
Zulkifli $^{1}$, Agustina Br Haloho ${ }^{1}$, Legiran ${ }^{2}$, Muhammad Ikhsan Kartawinata ${ }^{3 *}$ \\ ${ }^{1}$ Department of Anesthesiology and Intensive Therapy, Faculty of Medicine, Universitas Sriwijaya, Palembang, Indonesia \\ ${ }^{2}$ Department of Anatomy, Faculty of Medicine, Universitas Sriwijaya, Palembang, Indonesia \\ ${ }^{3}$ Specialized Residency Training, Department of Anesthesiology and Intensive Therapy, Faculty of Medicine, Universitas \\ Sriwijaya, Palembang, Indonesia
}

*Corresponding author:

Muhammad Ikhsan Kartawinata

Specialized Residency Training, Department of Anesthesiology and Intensive Therapy, Faculty of Medicine, Universitas Sriwijaya, Palembang, Indonesia

Email:

ikhsan.kartawinata@gmail.com

https://doi.org/10.37275/jacr.v2i2.161

\begin{abstract}
Introduction. The aerosol box can reduce the risk of droplet and aerosol transmission from the patient to the operator when performing intubation, but in practice, an aerosol box makes the glottis visualization less evident, and the operator moves less space with less space the aerosol box. This study aimed to compare ETT duration using an aerosol box and without an aerosol box using a video laryngoscope.

Methods. This study was a clinical trial with a post-test-only control design. The study was carried out from February 2021 to May 2021 at the Central Operating Theater of dr. Mohammad Hoesin General Hospital Palembang. The sample in this study was all patients who underwent elective surgery under general anesthesia using intubation at the Central Surgical Installation of dr. Mohammad Hoesin Palembang. After the data is collected, it is analyzed using the SPSS 22.0 program with the appropriate test.
\end{abstract}

Results. There were no differences in subject characteristics (age, sex, BMI, Mallampati score, TMD, Cormack Lehane, limited mouth opening, short neck, limited mouth movement) between the group using the aerosol box and the group without the aerosol box. The duration of intubation without an aerosol box is $30.67+2.63$ seconds, and using an aerosol box is $44.53+2.89$ seconds. There was a significant difference between the two groups in the duration of patient intubation ( $\mathrm{p}<0.001)$. However, there was no significant difference in complications in the two groups ( $p>0.05$ ).

Conclusion. The duration of the endotracheal tube insertion using an aerosol box is more extended than without an aerosol box in elective surgery patients.

Keywords: Intubation, aerosol box, COVID-19, video laryngoscope, personal protective equipment. 


\section{Introduction}

Endotracheal intubation is routine airway management performed by anesthesiologists. Endotracheal intubation procedure is not without risk; many complications can occur during the procedure, such as trauma to the airway mucosa, difficulty in intubating, and serious complications. On March 11, 2020, the World Health Organization (WHO) declared that the coronavirus disease 2019 (COVID-19) had become a worldwide pandemic. The severe acute respiratory syndrome-corona virus-2 (SARS-CoV-2) that causes COVID-19 is highly contagious, and the highest viral load of SARS-CoV-2 appears in the sputum and upper respiratory tract secretions. Airway management procedures, including tracheal intubation, can generate aerosols that increase the risk of transmission. ${ }^{1-2}$

The COVID-19 pandemic poses a greater risk to health workers, as shown by the increasing number of deaths of health workers in many countries. The main challenge for intubating patients with COVID-19 is the operator's exposure to aerosols and droplets from the patient. Therefore, consideration of the anesthesiologist to increase safety during laryngoscopy and intubation requires personal safety equipment. Several recent studies suggest aerosol boxes and level 3 PPE reduce the risk of transmission of droplets and aerosols from patient to operator during intubation. However, in practice, the use of level 3 PPE makes glottis visualization less clear, and operator movement space is reduced in the presence of an aerosol box..$^{3-}$ 5

Video laryngoscope (LV) will increase the visual field of laryngeal structures compared to direct visualization using direct laryngoscopy (LD). The angle of view created using a video laryngoscope is displayed on the monitor, so eye-hand coordination with the viewing angle needs to be directed at the monitor. ${ }^{6}$ The Anesthesia Patient Safety Foundation (APSF) and the American Society of Anesthesiologists (ASA) recommend using a video laryngoscope to insert an ETT in COVID-19 patients because it can minimize failure and shorten the time required. ${ }^{7,8}$ This study was aimed to compare of the duration of ETT insertion using an aerosol box with no aerosol box when performing intubation procedures using a video laryngoscope in elective surgery patients at RSMH Palembang.

\section{Methods}

This research is an experimental/clinical trial with a post-test-only control design. This study compared the duration of endotracheal tube insertion using an aerosol box with no aerosol box by assessing the duration of intubation after manipulation/intervention of the intubation procedure. The study population 


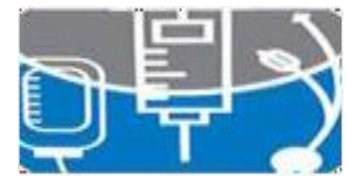

\section{Journal of Anesthestology \& \\ ClinicalResearch}

was all patients who underwent elective surgery under general anesthesia using intubation at dr. Mohammad Hoesin Palembang in February until the number of samples is met. The unpaired numerical, analytical sample size formula determined the research sample size. By paying attention to dropouts and withdrawals of $10 \%$, the sample size for each group is 40 subjects, so the total sample size is 80 subjects. The samples were divided into two groups, group A underwent endotracheal intubation using an aerosol box, and group B underwent intubation without using an aerosol box. Randomization was carried out using block randomization, namely by dividing groups per block consecutively, then the researcher compiled a table of random numbers and determined the treatment to be carried out in each of these blocks.

Inclusion criteria in this study were patients undergoing elective surgery with general anesthesia intubation, ages between 18 years to 65 years, ASA Physical status I/II, Mallampati I/II criteria, TMD 6 $\mathrm{cm}$, body mass index $18.5-30 \mathrm{~kg} / \mathrm{m} 2$. At the same time, the exclusion criteria were patients who refused to be included in this study. Withdrawal criteria were patients who withdrew from the study. Drop out criteria are patients who experience dangerous complications during induction of anesthesia or intubation, and intubation takes more than 3 minutes or more than two intubation attempts.

Operators are performing intubation using PPE level 3 (head cap, N95 1860 mask, surgical mask, isolation gown, disposable latex gloves, goggles, face shield, and full head hood). Before intubation, attach a monitor (blood pressure, ECG, SpO2) and position the patient supine with the head supported using a pillow as high as $10 \mathrm{~cm}$. Adjust the height of the operating table so that the patient's head is at the waist level of the operator who will perform intubation, then an aerosol box is installed in the study using an aerosol box. The patients were pre-oxygenated for 3 minutes; then anesthesia was induced by the operator assistant (resident with green level competence). Induction was started by giving midazolam $10 \mu \mathrm{g} / \mathrm{KgBW}$, fentanyl $1 \mu \mathrm{g} / \mathrm{KgBW}$, and propofol $1.5 \mathrm{mg} / \mathrm{kg}$ BW. After the study subjects fell asleep, positive pressure ventilation was performed using a facemask with $100 \%$ oxygen and maintenance of anesthetic sedation using the inhalation anesthetic sevoflurane 1 MAC. If the research subject can be ventilated using a facemask, continued with $0.5 \mathrm{mg} / \mathrm{kg} \mathrm{BW}$ atracurium. If the research subject cannot be ventilated using a facemask, then the LMA is installed. Research subjects are considered to drop out. After 3 minutes after administering atracurium $0.5 \mathrm{mg} / \mathrm{kg} \mathrm{BW}$, laryngoscopy was performed without compression of the cricoid cartilage using a video laryngoscope to view the visualization of the glottis performed by the study participants, after which the endotracheal tube was intubated and then cuff inflated $5 \mathrm{ml}$ of air at the time of intubation. The ETT is confirmed by auscultation of both lungs and the appearance of waves and numbers on a capnographic monitor or ETCO2 (end-tidal $\mathrm{CO}_{2}$ ). The duration of ETT insertion, number of attempts 


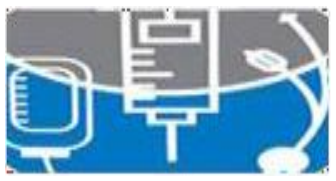

\section{Journal of Anesthesiofogy \& \\ Clinical Research}

at intubation, hemodynamics (blood pressure, heart rate, $\mathrm{SpO}_{2}$ ). If the patient had difficulty intubating, where more than two attempts to insert an endotracheal tube or the intubation time reached 3 minutes, the patient was considered dropping out.

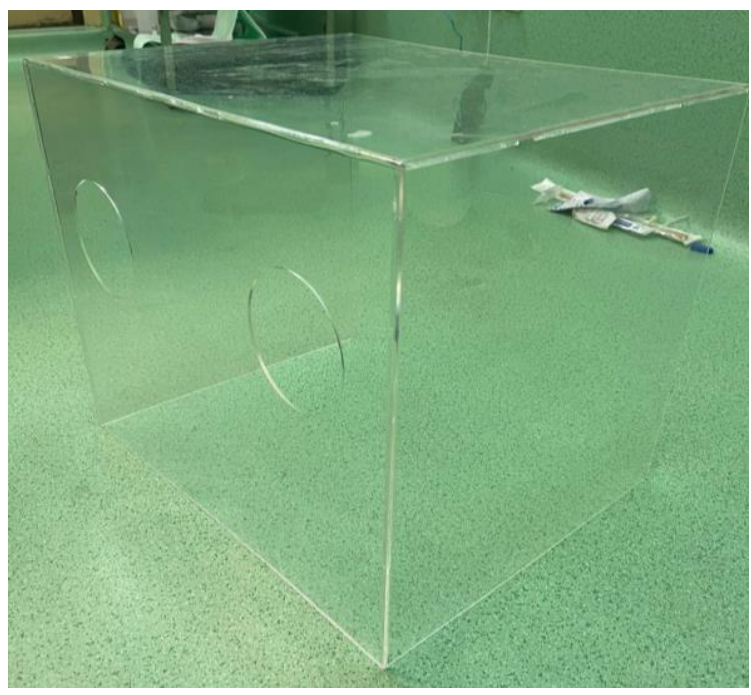

Figure 1. Aerosol box

The data taken is by the variables studied from the characteristics of the subject and the main research variables. Descriptive analysis is presented in the form of graphic tables and narratives. Numerical data are presented in the form of proportions, mean \pm standard deviation, median, minimum and maximum values. Categorical variables will be displayed in the form of frequency distributions and percentages.

Data on the duration of ETT insertion will be compared between groups intubated using an aerosol box and without an aerosol box numerically. Then the complication and confounding data will be analyzed statistically according to the data type. Chi-Square or Fisher's Exact test was used to analyze categorical data. $\mathrm{P}<0.05$ decided the criteria for accepting and rejecting the hypothesis.

\section{Results}

Sampling was carried out from February 2021 to May 2021, obtained 82 research subjects who met the inclusion criteria, but two subjects refused to participate in the study so that there were a total of 80 research subjects. The 80 subjects were then randomized and divided into two groups: the intubation group using an aerosol box and the intubation group without using an aerosol box. Each group consisted of 40 subjects, and there were no dropouts in this study. The general characteristics of research subjects between the two groups according to age, sex, BMI, Mallampati, TMD, Cormack Lehane, limited mouth opening, 

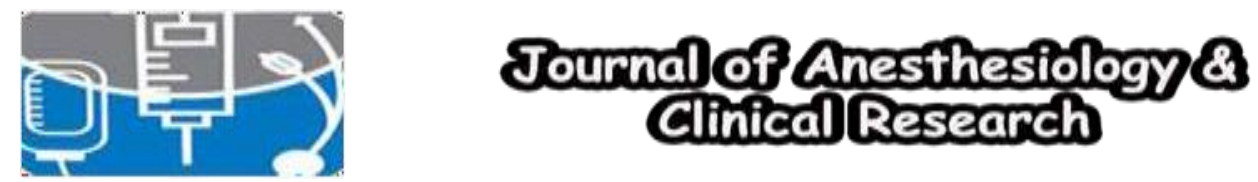

neck length, and limited neck movement did not show significant differences ( $p>0.05$; table 1).

Table 1. Characteristics of research subjects

\begin{tabular}{|c|c|c|c|}
\hline \multirow{2}{*}{ Variable } & \multicolumn{2}{|c|}{ Aerosol box } & \multirow[t]{2}{*}{$\mathrm{p}$} \\
\hline & No & Yes & \\
\hline Age, mean \pm SD & $42.25 \pm 13.77$ & $42.13 \pm 13.42$ & 0,967 \\
\hline $\begin{array}{l}\text { Gender, } \mathrm{n}(\%) \\
\text { Male } \\
\text { Female }\end{array}$ & $\begin{array}{l}16(40.0 \%) \\
24(60.0 \%)\end{array}$ & $\begin{array}{l}11(27.5 \%) \\
29(72.5 \%)\end{array}$ & 0,344 \\
\hline $\begin{array}{l}\text { BMI, n (\%) } \\
\text { Normal } \\
\text { Overweight } \\
\end{array}$ & $\begin{array}{c}32(80.0 \%) \\
8(20.0 \%)\end{array}$ & $\begin{array}{c}36(90.0 \%) \\
4(10.0 \%)\end{array}$ & 0,210 \\
\hline $\begin{array}{l}\text { Mallampati score, n (\%) } \\
1 \\
2 \\
\end{array}$ & $\begin{array}{c}37(92.5 \%) \\
3(7.5 \%) \\
\end{array}$ & $\begin{array}{c}35(87.5 \%) \\
5(12.5 \%) \\
\end{array}$ & 0,712 \\
\hline $\begin{array}{l}\text { TMD, } \mathrm{n}(\%) \\
\geq 6.5 \mathrm{~cm} \\
<6.5 \mathrm{~cm}\end{array}$ & $\begin{array}{c}40(100.0 \%) \\
0(0.0 \%)\end{array}$ & $\begin{array}{c}38(95.0 \%) \\
2(5.0 \%)\end{array}$ & 0,494 \\
\hline \multirow{2}{*}{ Variable } & \multicolumn{2}{|c|}{ Aerosol box } & $\mathrm{p}$ \\
\hline & No & Yes & \\
\hline $\begin{array}{l}\text { Cormack Lehane, n (\%) } \\
\text { I } \\
\text { II }\end{array}$ & $\begin{array}{l}29(72.5 \%) \\
11(27.5 \%) \\
\end{array}$ & $\begin{array}{l}27(67.5 \%) \\
13(32.5 \%) \\
\end{array}$ & 0,626 \\
\hline $\begin{array}{l}\text { Limited mouth opening, } \mathrm{n}(\%) \\
\geq 3 \text { finger } \\
<3 \text { finger }\end{array}$ & $\begin{array}{c}40(100 \%) \\
0(0.0 \%)\end{array}$ & $\begin{array}{c}40(100 \%) \\
0(0.0 \%)\end{array}$ & 1,000 \\
\hline $\begin{array}{l}\text { Neck length, n (\%) } \\
\quad<7 \mathrm{~cm} \\
7-9 \mathrm{~cm}\end{array}$ & $\begin{array}{c}9(22.5 \%) \\
31(77.5 \%)\end{array}$ & $\begin{array}{l}10(25.0 \%) \\
30(75.0 \%)\end{array}$ & 0,793 \\
\hline $\begin{array}{l}\text { Limited neck movement, n (\%) } \\
\text { No } \\
\text { Yes }\end{array}$ & $\begin{array}{c}40(100 \%) \\
0(0.0 \%)\end{array}$ & $\begin{array}{c}40(100 \%) \\
0(0.0 \%)\end{array}$ & 1,000 \\
\hline
\end{tabular}

Table 2 shows that the duration of intubation without using an aerosol box is $30.67+2.63$ seconds and intubation using an aerosol box is $44.53+2.89$. Intubation duration data were normally distributed after being analyzed by the Kolmogorov-Smirnov test $(\mathrm{p}=0.200)$. In the independent $\mathrm{t}$-test analysis, it was found that there was a significant difference between the duration of intubation using an aerosol box or without an aerosol box with a p-value of $<0.001$. 


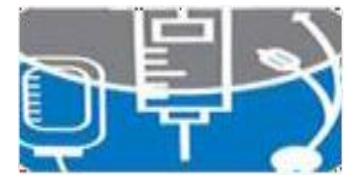

\section{Journal of Anesthesiology \& \\ Clinical Research}

Table 2. Comparison of the duration of intubation using an aerosol box and without an aerosol box

\begin{tabular}{|c|c|c|c|}
\hline \multirow{2}{*}{ Variable } & \multicolumn{2}{|c|}{ Aerosol box } & \multirow{2}{*}{ p } \\
\cline { 2 - 3 } & No & Yes & $<0.001$ \\
\hline Duration of intubation (second) & $30.67 \pm 2.63$ & $44.53 \pm 2.89$ & $<$ \\
Min.- max. (second) & $25,9-37,1$ & $38,9-50,1$ & \\
\hline
\end{tabular}

Note: Test using independent t-test, $\mathrm{p}<0.05=$ significant

The comparison of the frequency of intubation using an aerosol box and without an aerosol box can be seen in table 3. It was found that in the intubation group without using an aerosol box, all study subjects were successfully intubated in the first experiment. In contrast, three patients were successfully intubated in the second experiment in the intubation group using an aerosol box. In the Fisher's Exact Test analysis test, it was found that there was no significant difference between intubation experiments using an aerosol box or without an aerosol box with a p-value of 0.241 .

Table 3. Comparison of the frequency of intubation using an aerosol box and without an aerosol box

\begin{tabular}{|c|c|c|c|c|}
\hline \multirow{2}{*}{$\begin{array}{c}\text { Frequency } \\
\text { intubation }\end{array}$} & \multicolumn{2}{|c|}{ Aerosol box } & p & $\begin{array}{c}\text { Odds ratio } \\
\text { (95\% CI) }\end{array}$ \\
\cline { 2 - 5 } & No & Yes & & 0,481 \\
\hline 1 & $40(100 \%)$ & $37(92.5 \%)$ & 0,241 & $(0,381-0,606)$ \\
\hline 2 & $0(0 \%)$ & $3(7.5 \%)$ & & \\
\hline
\end{tabular}

Analysis using Fisher's Exact Test, $\mathrm{p}>0.05=$ not significantly related.

The relationship of complications of intubation between using an aerosol box and without using an aerosol box can be seen in table 4. The table shows that no complications were found without the use of an aerosol box. Meanwhile, in the intubation group using an aerosol box, 3 (7.5\%) subjects experienced complications of lip injury, and $2(5 \%)$ subjects experienced hypoxia complications, with saturation reaching $92 \%$. In the chi-square analysis test, it was found that there was no significant relationship between the use of an aerosol box or without an aerosol box on the emergence of complications with a p-value of 0.055 . 

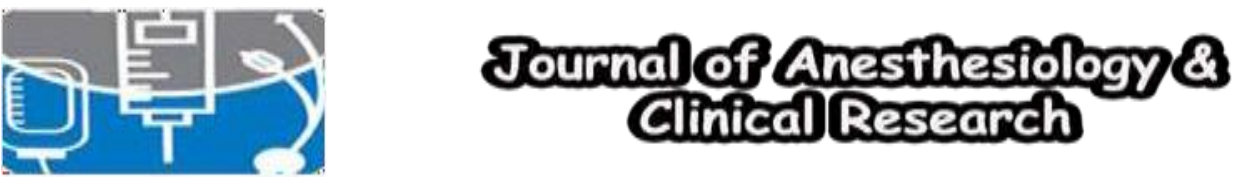

Table 4. The relationship of complications intubation using an aerosol box and without an aerosol box

\begin{tabular}{|c|c|c|c|c|}
\hline \multirow{2}{*}{ Complications } & \multicolumn{2}{|c|}{ Aerosol box } & p & Odds ratio \\
\cline { 2 - 3 } & No & Yes & & (95\% CI) \\
\hline Yes & $0(0 \%)$ & $5(12.5 \%)$ & \multirow{2}{*}{0,055} & 0,467 \\
\hline No & $40(100 \%)$ & $35(87.5 \%)$ & & $(0,366-0,594)$ \\
\hline
\end{tabular}

Analysis using Fisher's Exact Test, $\mathrm{p}>0.05=$ not significantly related.

\section{Discussion}

Endotracheal tube insertion is the highest risk of contamination aerosol in airway management. Several studies have mentioned the benefits of a barrier or, in this case, the use of an aerosol box to prevent infection transmission during endotracheal intubation. ${ }^{6-8}$ However, some anesthesiologists admit to having difficulty using the aerosol box even though experienced assistants have assisted them.

The distribution of age, gender, BMI, TMD, Cormack Lehane, limited mouth opening, neck length, and limitation of neck movement to the use of aerosol box were comparable ( $>0.05)$. Thus, based on Fisher's Exact analysis, it was found that there was no significant difference between the distribution of age, gender, BMI, TMD, Cormack Lehane, limited mouth opening, neck length, and limited mouth movement on the use of aerosol box so that the sample was homogeneous and ideal for conducting the study.

Table 2 shows that the duration of intubation without using an aerosol box is 30.67 seconds, and using an aerosol box is 44.53 seconds. In the independent t-test analysis, it was found that there was a significant difference between the duration of intubation using an aerosol box and without an aerosol box with a pvalue of $<0.001$. The use of an aerosol box in the intubation procedure has been shown to increase the duration of the intubation procedure. ${ }^{9}$ However, some literature mentions the benefits of protection and a reduction in the risk of contamination with the use of appropriate personal protective equipment along with the use of an aerosol box. This is in line with the research of Begley et al., who found that the average intubation with the aerosol box was 10 seconds longer than without the aerosol box (52.4 seconds compared to 42.9 seconds). ${ }^{10}$ Based on the results of our 45 interviews with operators who perform intubation in this study, several factors can cause the use of aerosol boxes to have a longer duration of intubation. Some of these factors are: limited movement of the operator in the aerosol box, the intubation device often hits the aerosol box, requires an assistant to fix the box position so that the box does not move, is not familiar with using a video laryngoscope, is not familiar with using an aerosol box, and poor glottis visualization due to 


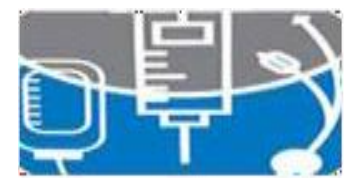

\section{Journal of Anesthesiology \& \\ Clinical Research}

dew on the video laryngoscope camera.

In the study, Wakabayashi et al. found that $80 \%$ of operators also complained of fogging on Google or operator glasses. ${ }^{5}$ The aerosol box makes viewing the glottis significantly more difficult, slowing down the endotracheal tube insertion time. This time difference is quite significant, plus the use of aerosol boxes can damage PPE, thereby increasing the risk of aerosol contamination for medical personnel, and the use of assistive devices such as boogies is also not possible in the box. Clinicians must weigh the benefits and risks of using aerosol boxes in daily practice. ${ }^{11-13}$

In table 3 , it is found that the frequency of intubation using the aerosol box as many as $3(7.5 \%)$ subjects failed in the first attempt of intubation, while in the intubation group without using an aerosol box, all subjects $(100 \%)$ were successfully intubated in the first experiment. The intubation failure in the first experiment in this study was due to the operator's lack of habit in using a video laryngoscope, the difficulty of operator movement when using level 3 PPE, coupled with the presence of an aerosol box. This result is in line with the study by Azhar et al., who found that 5.4\% of patients were successfully intubated after the second trial in the aerosol box group compared to the group without using the aerosol box, all of which were successful in the first experiment. ${ }^{7}$

In table 4 , it is found that the comparison of the incidence of complications in the group using the aerosol box and without using the aerosol box does not have a significant relationship with the $\mathrm{p}$-value $=$ 0.055. This is in line with the research conducted by Wakabayashi et al., who said that the use of aerosol boxes does not significantly affect the duration of intubation, the success of intubation, and the complications that arise when performed by a trained anesthesiologist. ${ }^{5}$ It can be concluded here that the use of aerosol boxes is not significantly related to the presence of complications, so its use as personal protective equipment in the current pandemic condition is quite effective.

The limitation of this research is that it was conducted in a single center in one hospital, so it is not easy to describe the general description of anesthesiology operators in Indonesia. This study also did not assess the effectiveness of aerosol boxes in preventing transmission of the COVID-19 virus. Aerosol box designs and aerosol box sizes can also differ between hospitals, so this study's results cannot be used in other aerosol box designs and sizes. This study was also not conducted in patients with a BMI > 35, where airway management may be more difficult in obese subjects, and the use of aerosol boxes may also be complex due to the small size of the aerosol box. 


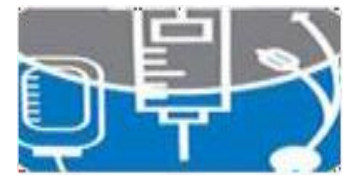

\section{Journal of Anesthesiology \& \\ Clinical Research}

\section{Conclusion}

There was a significant difference in the duration of endotracheal Tube insertion using an aerosol box with no aerosol box in elective surgery patients during the COVID-19 pandemic $(\mathrm{p}<0.001)$. Aerosol boxes can be used in elective surgery patients during the pandemic; although the duration of insertion of the endotracheal tube is long, there is no significant difference in complications between the group with the aerosol box and without the aerosol box.

\section{References}

1. Saito T, Taguchi A. Asai T. Videolaryngoscopy for tracheal intubation in patients with COVID-19. Br J Anaesth. 2020; 1(1): 283-6.

2. Aletreby, Waleed Th, Madi AF, Ramadan OE, Alodat MA, Huawit BM. Impact of aerosol box on duration of intubation of COVID-19 patients: simulation cross-over study. IJHSR. 2020; 10(7): 227.

3. Brown EN, Pavone KJ, Naranjo M. Multimodal general anesthesia: Theory and practice. Anesth Analg. 2018; 127(5): 1246-58.

4. Kearsley R. Intubation boxes for managing the airway in patients with COVID-19. Anaesthesia. 2020; 75: 962-77.

5. Wakabayashi R, Ishida T, Yamada T, Kawamata M. Effect of an aerosol box on tracheal intubation difficulty. J Anesth.2020; 34: 790-3.

6. Ye L, Wong DT, Liu J, Zhu T. Mallampati class does not affect the success rate of intubation through an intubating laryngeal mask airway with reverse tracheal tube direction. 2013 Mar; 79(3): 227-31.

7. Azhar MN, Bustam A, Poh K, Zahedi AZA, Nazri MZAM, Arifin MAA, et al. COVID-19 aerosol box as protection from droplet and aerosol contaminations in healthcare workers performing airway intubation: a randomised crossover simulation study. Emerg Med J. 2020; 38: 111-7.

8. Shin M, Bai SJ, Lee KY, Oh E, Kim HJ. Comparing McGRATH® MAC, C-MAC®, and Macintosh Laryngoscopes Operated by Medical Students: A Randomized, Crossover, Manikin Study. Biomed Res Int. 2016; 16(8): 1-8.

9. Feldman O, Samuel N, Kvatinsky N, Idelman R, Diamand R, Shavit I. Endotracheal intubation of COVID-19 patients by paramedics using a box barrier: A randomized crossover manikin study. Journal Pone. 2020; 16(3): 1-6. 


\section{Sournal of Anesthesiology \& \\ Clinical Research}

10. Begley JL, Lavery KE, Nickson CP, Brewster DJ. The aerosol box for intubation in corona virus dissease 2019 patients: an in-situ simulation crossover study. Anaesthesia. 2020;75: 1014-21.

11. Lim ZJ, Reddy MP, Karalapillai D, Shekar K, Subramaniam A. Impact of an aerosol box on time to tracheal intubation: systematic review and meta-analysis. Br J Anaesth. 2021 Mar; 126(3): 1225.

12. Madabhushi P, Kinthala S, Ankam A, Chopra N, Porter BR. Time to adapt in the pandemic era: a prospective randomized non-inferiorty study comparing time to intubate with and without the barrier box. BMC Anesthesiology. 2020; 20(1): 232.

13. Hagberg CA, Normand KC, Vargas LA, Burnett Jr A, Sridhar S, Cai C. Use of the McGRATH MAC: To view or not to view? Trends in Anaesthesia and Critical Care. 2018; 1-9. 